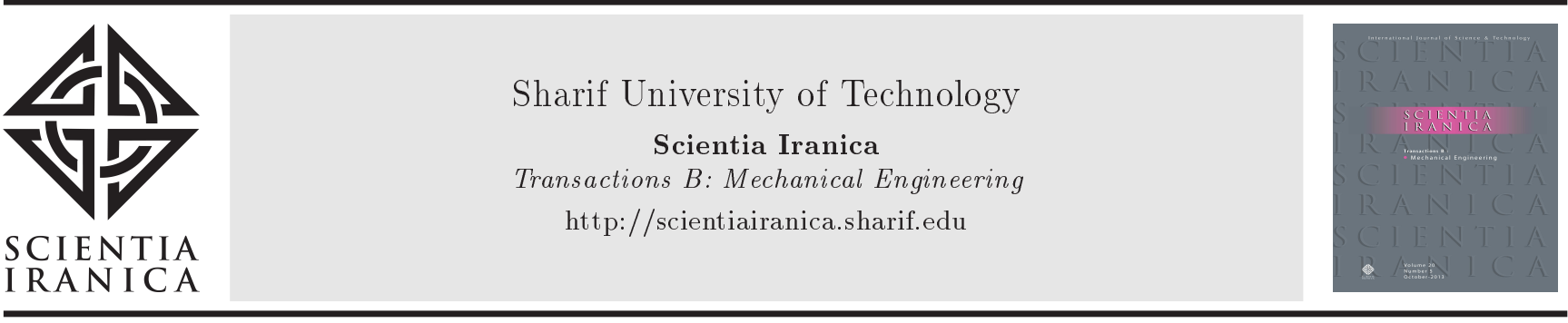

\title{
Modelling and optimization of robotic manipulator mechanism for computed tomography guided medical procedure
}

\author{
Sh. Kamlesh Shah and R. Mishra* \\ School of Mechanical Engineering, KIIT Deemed to be University, Bhubaneswar, 751024, India.
}

Received 2 December 2020; received in revised form 19 April 2021; accepted 5 July 2021

\author{
KEYWORDS \\ Genetic algorithm; \\ Link length \\ optimization; \\ Manipulator \\ workspace; \\ Error minimization; \\ Robotic manipulator.
}

\begin{abstract}
Although industrial robots are common, higher degree of manipulability might be required to expand the applications of manipulators in the field of medicine. Modifying the mechanical design of a robot as per the workspace can be perceived as an optimization problem. Hence, a novel spatial manipulator is designed for a diagnostic apparatus using different optimization algorithms. Standard Genetic Algorithm (SGA) and Genetic Algorithm (GA) with hybrid functions like Pattern Search (PS) and fmincon are proposed to optimize the link lengths of 3-Degree-Of-Freedom (DOF), 6-DOF, and novel 9-DOF hybrid redundant manipulators. A 9-DOF robot is designed to manipulate a needle in Computed Tomography (CT) machine environment. The fitness function for all the manipulators is formulated using forward kinematic equations according to their workspace. Limits and constraints of each link have been decided in advance. A comparative study between all the hybrid GA functions is performed. MATLAB is used to solve and train the proposed GA method for optimizing the link lengths. Results show that the GA with PS provide better-optimized link lengths for 3-DOF and 9-DOF manipulators while fmincon is well suited for a 6 -DOF robot manipulator. Workspace and dead zone analysis is also performed using the optimized link lengths obtained.
\end{abstract}

(C) 2022 Sharif University of Technology. All rights reserved.

\section{Introduction}

Infusion of robots has taken place in almost all the human life sectors, from manufacturing and household to hospitals. Among all the other types of robots, one of the most commonly used robots is robotic arm manipulator. Robotic arms are used in manufacturing industries for tasks like pick and place, painting, welding, etc., while they are used for surgical purposes in the medical field as well.

\footnotetext{
*. Corresponding author.

E-mail addresses: shubham.prasad1@gmail.com (Sh. Kamlesh Shah); rubymishrafme@kiit.ac.in (R. Mishra)
}

doi: $10.24200 /$ sci. 2021.57259 .5149
Industrial robots characterized by three and six Degrees Of Freedom (DOF) have remained widely applicable for many years. The standard 6-DOF robotic manipulator is sufficient for most applications in industrial and home environments. In some instances, redundancy and more DOF are required to achieve the desired target. Some of these applications include robots in medicine, reactors, space, underwater explorations, etc. For such applications that require higher DOF and redundancy, robotic manipulators with more than 6 DOFs are necessary. The first step in designing a robotic manipulator is its link lengths.

Calculations of link lengths for manipulators with more than 6 DOFs are computationally complex, making it difficult to reach just one solution. Development of a redundant manipulator is not as straightforward 
as that of a six or lower DOF manipulator. The main problem of finding the right solution to such issues is that they are multi-objective, multi-constrained, and multivariate. The optimization of parameters such as link length, total reach, and workspace is essential and often contradicting [1].

In the field of robotics, Genetic Algorithm (GA) has been used to optimize various tasks such as path planning of a 3-DOF manipulator to minimize the joint angle change while avoiding obstacles [2]. GA is also used to obtain a solution to inverse kinematics [3]. In the case of a 6-DOF manipulator, the parameter of the PID control system is optimized. This method also exhibited a better result than commonly used techniques such as the empirical approach and trial-error method [4]. To dynamically model a 9-DOF hydraulic manipulator, GA was used. Varying effects of the crossover method, crossover rate, and encoding scheme on the performance of the GA were also analyzed [5]. To reduce the error in reaching the target points of a heavy-duty hydraulic serial robotic manipulator, its structure needs to be optimized. Constraints are applied according to its underground tunnelling taskbased workspace. Link lengths were effectively optimized to minimize position and orientation errors [6].

Active research is being conducted to optimize the workspace reach, dexterity, and structure of the serial kinematic robotic manipulator. GA is used to optimize the topology of a modular robot, taking into consideration a specific task [7]. GA was also used to optimize link length and gearbox of a 3-DOF serial manipulator taking into account its dynamic model performance [8]. Optimization of link length, link diameter, and link thickness of a 6-DOF manipulator designed for cleaning a fish tank is also carried out using GA [9]. GA is very versatile and there are many different variants of GA that are used in robotics for optimization of various structural and design parameters. Multi-Objective Genetic Algorithm (MOGA), MultiObjective Differential Evolution (MODE), and elitist Non-dominated Sorting Genetic Algorithm (NSGA-II) are some of the modified GA used to optimize the design parameters of a robotic gripper [10]. Another variant of GA Multi-Objective Particle Swarm Optimization (MOPSO) is used to optimize the 3-DOF parallel manipulator design [11]. Topology optimization of the robotic link was also attempted using a generative design technique, which is a similar form of GA [12]. Attempt to optimize crane designs has also been made using GA. Cranes can also be considered as a robotic system [13]. Structural optimization of a planar 4-DOF robotic manipulator was obtained using GA to reach a target from a starting point without hitting obstacles. The manipulator had hybrid rotary and prismatic joints [14]. Another attempt is made to optimize the travel time and space of a 3-DOF planar manipulator using GA. The motion planning is optimized in such a way that the robot avoids collision with obstacles within the given torque limits [15]. The trajectory and torque profile of another 3-DOF micro-robot was optimized for surgery. Optimization completed GA, hybrid GA, Pattern Search (PS), and Particle Swarm Optimization (PSO) methods. All the optimization techniques performed almost the same besides hybrid GA, which was slightly better by $3 \%$ [16]. Attempts have also been made to design a robotic manipulator with 5-axis for application under Computed Tomography $(\mathrm{CT})$ guidance. Trajectory and path were generated in the same studies $[17,18]$. The trajectory of a biped robot is optimized for random obstacle avoidance using regression. Ultrasonic distance sensors are used as the sensory input for the regression controller, which then provides the heading angle for the biped robot. Industrial automation benefits from random obstacle avoidance research [19]. Even in the case of flexible robots, adaptive dynamic surface control is implemented to enhance its tracking performance. Parametric uncertainties are overcome using the firstorder derivative filter of the inertial parameters [20]. An SCARA robot manipulator is also modeled and simulated as a PRR-type manipulator. Link lengths and $\mathrm{DH}$ parameters of this manipulator are calculated mathematically using forward and inverse kinematic equations. The performance of its pick and place was then observed experimentally [21]. Path of a mobile robot is optimized using a GA to obtain a smoother and shorter trajectory. Adaptive penalty factor is used in GA for ensuring the safety of the robot during obstacle avoidance [22]. Trajectory optimization of a $6-\mathrm{DOF}$ manipulator using GA, random average recombination, differential evolution, and linear and geometric cooling strategy was attempted. The goal of this optimization is torque minimization, while a 6 -DOF robot is carrying a load from point to point. GA achieved the best result in torque minimization [23]. To minimize the cycle time and vibration of a 6-DOF robot for electronic industries, GA was implemented. It increases the productivity of the electronic PCB manufacturing industry and reduces vibrations by optimizing the velocity of the robot assembly [24]. Much focus has been given to trajectory optimization using GA, regression, and various other algorithms, but research on the optimization of $\mathrm{DH}$ parameters based on the required workspace for standard and redundant manipulators has been lacking. Limited research has been conducted for link length optimization of a robot for application in the medical field.

Similar attempts have been made to optimize the structure and trajectory of various DOF robotic manipulators, but there is a limited study on the comparison of link length optimization for the desired workspace reach using GA for 3-DOF, 6-DOF, and 9- 
DOF robot manipulators in CT machine environment. Manipulating robots in a CT environment poses quite a challenge, as the body of the CT machine is a major obstacle. The scanning area is the workspace of any manipulator working with a CT machine. The diameter of the scanning region of a standard CT machine is $70 \mathrm{~cm} \mathrm{[25];} \mathrm{hence,} \mathrm{the} \mathrm{robotic} \mathrm{manipulator}$ must reach the scanning area and maneuver in that region without touching the body of the CT machine.

The novelty of this research is the optimization of link lengths of 3-DOF, 6-DOF, and 9-DOF robotic manipulators. 9-DOF robot is a novel manipulator specifically designed to be mounted on the CT bed with an arc design. Optimization is carried out to determine a better set of link lengths so that the robots can reach and maneuver in the desired three-dimensional CT workspace. Only forward kinematics and error function are used as fitness functions in GA and GA with hybrid functions to optimize the $\mathrm{DH}$ parameters including link lengths and joint displacement. Different types of GA coupled with hybrid functions like PS and fmincon are compared for all three robotic manipulators. Different hybrid functions are modified to select the better optimized set of link lengths and joint displacements for each robot.

\section{Manipulator designs and DH parameters}

Standard rotary 3-DOF, rotary 6-DOF, and a novel 9-DOF hybrid robotic manipulators are designed for this research. The kinematic model of all the robotic manipulators is designed in MATLAB. DH parameters of all the robot manipulator are also determined and mentioned in the respective tables. The red items are the end effector link and the joints including rotary and prismatic, while the blue items denote the rest of the links of the robotic manipulators.

Figure 1 shows the kinematic model of a 3-DOF robot manipulator with rotary joints. The workspace of this robot is spherical when all the joints have full $360^{\circ}$ motion.

$\mathrm{DH}$ parameters of the 3-DOF manipulator are shown in Table 1 where $i$ signifies the link number. It is observed that the robot is a standard rotary 3-DOF manipulator.

Figure 2 shows the kinematic model of a 6 -DOF

Table 1. DH parameters of the 3-DOF robot manipulator.

\begin{tabular}{ccccc}
\hline $\begin{array}{c}\text { Joint } \\
\text { number }\end{array}$ & $\begin{array}{c}\text { Link } \\
\text { length } \\
\left(\boldsymbol{l}_{\boldsymbol{i}}\right)\end{array}$ & $\begin{array}{c}\text { Joint } \\
\text { displacement } \\
\left(\boldsymbol{d}_{\boldsymbol{i}}\right)\end{array}$ & $\begin{array}{c}\text { Link } \\
\text { twist } \\
\left(\boldsymbol{\alpha}_{\boldsymbol{i}}\right)\end{array}$ & $\begin{array}{c}\text { Joint } \\
\text { angle } \\
\left(\boldsymbol{\theta}_{\boldsymbol{i}}\right)\end{array}$ \\
\hline Joint 1 & 0 & $d_{1}$ & $\pi / 2$ & $\theta_{1}$ \\
Joint 2 & $l_{2}$ & 0 & 0 & $\theta_{2}$ \\
Joint 3 & $l_{3}$ & 0 & 0 & $\theta_{3}$ \\
\hline
\end{tabular}

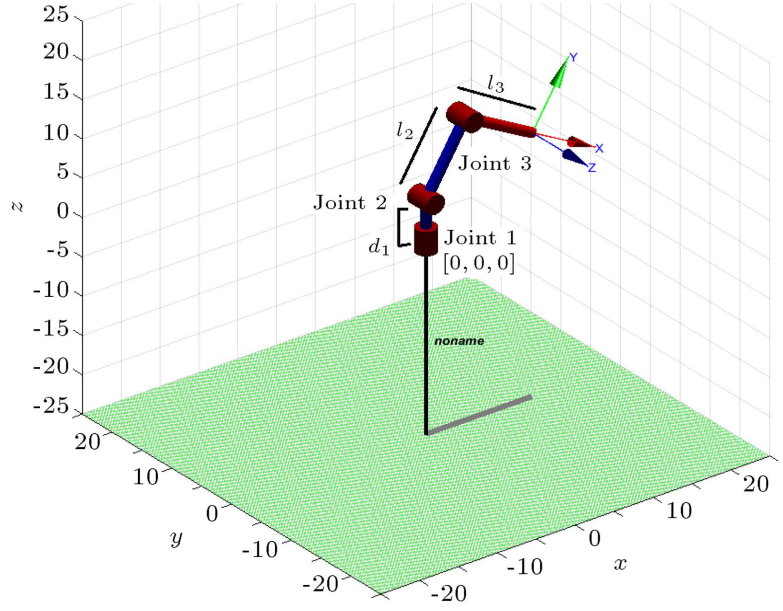

Figure 1. Kinematic model of a 3-DOF robotic manipulator.

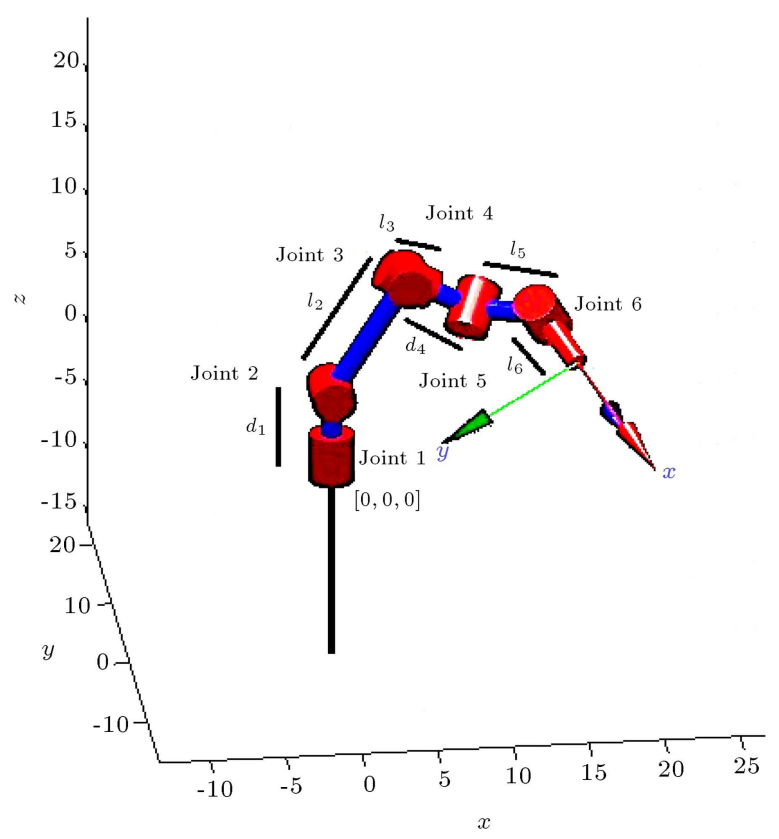

Figure 2. Kinematic model of a 6 -DOF robotic manipulator.

robot manipulator with rotary joints. The workspace of this robot is very versatile as it has freedom of motion in every direction and orientation.

DH parameters of the 6-DOF manipulator are shown in Table 2. It is observed that the robot is a standard rotary 6-DOF manipulator.

Figure 3(a) shows the CAD and kinematic model of a novel 9-DOF robot manipulator with rotary and prismatic joints. This robot is designed to be mounted on the CT bed and maneuver in the scanning area. Figure 3(a) shows a CAD model of the robotic manipulator mounted on a CT bed and Figure 3(b) shows the kinematic model of the manipulator. In Figure 3, all the joints of the robotic manipulator are shown at $0^{\circ}$ configuration. 


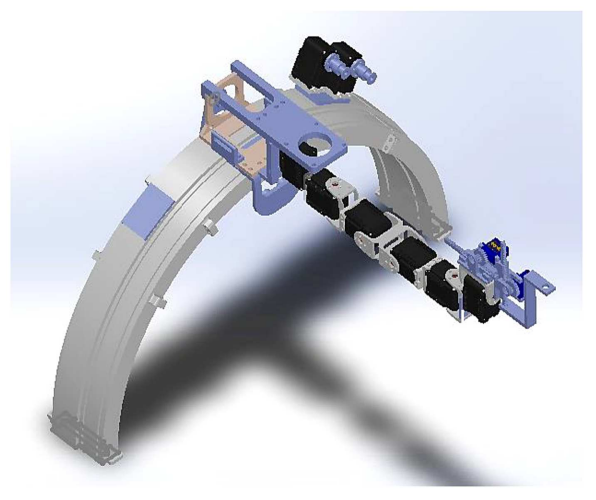

(a)

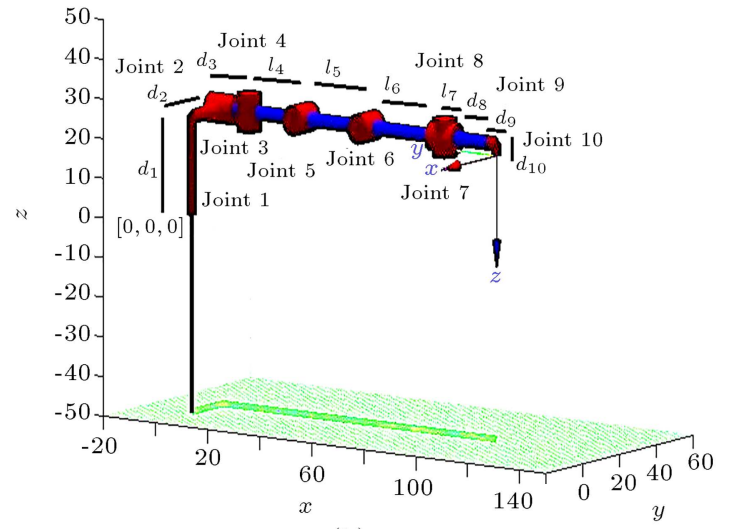

(b)

Figure 3. (a) CAD of the novel 9-DOF robotic manipulator. (b) Kinematic model of the novel 9-DOF robotic manipulator.

Table 2. DH parameters of the 6-DOF robot manipulator.

\begin{tabular}{ccccc}
\hline $\begin{array}{c}\text { Joint } \\
\text { number }\end{array}$ & $\begin{array}{c}\text { Link } \\
\text { length } \\
\left(\boldsymbol{l}_{\boldsymbol{i}}\right)\end{array}$ & $\begin{array}{c}\text { Joint } \\
\text { displacement } \\
\left(\boldsymbol{d}_{\boldsymbol{i}}\right)\end{array}$ & $\begin{array}{c}\text { Link } \\
\text { twist } \\
\left(\boldsymbol{\alpha}_{\boldsymbol{i}}\right)\end{array}$ & $\begin{array}{c}\text { Joint } \\
\text { angle } \\
\left(\boldsymbol{\theta}_{\boldsymbol{i}}\right)\end{array}$ \\
\hline Joint 1 & 0 & $d_{1}$ & $\pi / 2$ & $\theta_{1}$ \\
Joint 2 & $l_{2}$ & 0 & 0 & $\theta_{2}$ \\
Joint 3 & $l_{3}$ & 0 & $-\pi / 2$ & $\theta_{3}-\pi / 2$ \\
Joint 4 & 0 & $d_{4}$ & $\pi / 2$ & $\theta_{4}$ \\
Joint 5 & $l_{5}$ & 0 & $\pi / 2$ & $\theta_{5}+\pi / 2$ \\
Joint 6 & $l_{6}$ & 0 & 0 & $\theta_{6}$ \\
\hline
\end{tabular}

DH parameters of the 9-DOF manipulator are given in Table 3 . It is observed that the robot is a redundant hybrid 9-DOF manipulator. The arc design of the manipulator constitutes 1-DOF. Arc design is developed for the ergonomics of the patient in a CT machine environment.

\section{Mathematical formulation}

GA is a heuristic approach to solving an optimization problem [26]. Here, the error functions are minimized to derive the optimum set of link lengths. GA needs a fitness function to optimize these link lengths and for a robotic manipulator, forward kinematic equations serve as a good fitness function because forward kinematic equations relate the $\mathrm{DH}$ parameters to the realworld workspace in $X, Y$, and $Z$ directions.

The transformation matrix is used to derive the forward and inverse kinematic equations. Mathematical equations for link lengths are derived from the kinematic equations. From these equations, a fitness function for obtaining the link lengths by training the GA is derived. In Eq. (1), shown in Box I, the general

Table 3. DH parameters of the 9-DOF robot manipulator.

\begin{tabular}{lccll}
\hline Joint number & $\begin{array}{c}\text { Link length } \\
\left(\boldsymbol{l}_{\boldsymbol{i}}\right)\end{array}$ & $\begin{array}{c}\text { Joint displacement } \\
\left(\boldsymbol{d}_{\boldsymbol{i}}\right)\end{array}$ & $\begin{array}{c}\text { Link twist } \\
\left(\boldsymbol{\alpha}_{\boldsymbol{i}}\right)\end{array}$ & $\begin{array}{c}\text { Joint angle } \\
\left(\boldsymbol{\theta}_{\boldsymbol{i}}\right)\end{array}$ \\
\hline Joint 1 (vertical) & $l_{1}$ & $d_{1}$ & $-\pi / 2$ & 0 \\
Joint 1 (horizontal) & $l_{2}$ & $d_{2}$ & $-\pi / 2$ & $-\pi / 2$ \\
Joint 2 & 0 & $d_{3}$ & $-\pi / 2$ & $\theta_{3}-\pi / 2$ \\
Joint 3 & $l_{4}$ & 0 & $-\pi / 2$ & $\theta_{4}-\pi / 2$ \\
Joint 4 & $l_{5}$ & 0 & 0 & $\theta_{5}$ \\
Joint 5 & $l_{6}$ & 0 & $-\pi / 2$ & $\theta_{6}$ \\
Joint 6 & $l_{7}$ & 0 & $\pi / 2$ & $\theta_{7}+\pi / 2$ \\
Joint 7 & 0 & $d_{8}$ & 0 & $\theta_{8}$ \\
Joint 8 & 0 & $d_{9}$ & 0 & 0 \\
Joint 9 & 0 & $d_{10}$ & 0 & 0 \\
\hline
\end{tabular}




$$
T_{(n-1)}^{n}=\begin{array}{cccc}
\cos \left(\theta_{n}\right) & -\sin \left(\theta_{n}\right) \times \cos \left(\alpha_{n}\right) & \sin \left(\theta_{n}\right) \times \sin \left(\alpha_{n}\right) & l_{n} \times \cos \left(\theta_{n}\right) \\
\sin \left(\theta_{n}\right) & \cos \left(\theta_{n}\right) \times \cos \left(\alpha_{n}\right) & -\cos \left(\theta_{n}\right) \times \sin \left(\alpha_{n}\right) & l_{n} \times \sin \left(\theta_{n}\right) \\
0 & \sin \left(\alpha_{n}\right) & \cos \left(\alpha_{n}\right) & d_{n} \\
0 & 0 & 0 & 1
\end{array}
$$

Box I

form of the transformation matrix used for all threerobot manipulators [27] is shown. ' $n$ ' denotes the number of joints in a robot manipulator. The units of all the link lengths and joint displacements are in $\mathrm{cm}$ and the link twist and joint angles are in degrees.

For a 3-DOF manipulator, Eq. (2) shows its transformation matrix formula:

$$
T_{0}^{3}=T_{0}^{1} \times T_{1}^{2} \times T_{2}^{3},
$$

Eqs. (3) to (5) show the forward kinematic equations of the 3-DOF robot:

$$
\begin{aligned}
& X_{1}=C_{1} *\left(l_{3}^{*} C_{23}+l_{2}{ }^{*} C_{2}\right), \\
& Y_{1}=S_{1}{ }^{*}\left(l_{3}{ }^{*} C_{23}+l_{2}{ }^{*} C_{2}\right), \\
& Z_{1}=d_{1}+l_{3} * S_{23}+l_{2} * S_{2},
\end{aligned}
$$

where $X_{1}, Y_{1}$, and $Z_{1}$ are the derived coordinates in the workspace of the 3 -DOF robot [28]. Here, $C_{n}$ and $S_{n}$ are cosine and sine of the $n$th joint, respectively, where $n=1,2, \cdots, n . C_{23}$ and $S_{23}$ represent cosine $\left(\theta_{2}+\theta_{3}\right)$ and sine $\left(\theta_{2}+\theta_{3}\right)$, respectively.

\subsection{Fitness function for 3-DOF robot \\ 3.1.1. Constraints}

In Eqs. (3) to (5) the link length equations of the 3DOF mahipulator are shown. It is observed that all the equations are interdependent, and it is not easy to come to a definitive solution to a range of target coordinate values. Hence, to find an optimum solution, GA is used. Error $_{1}$ shows the equation for error between all the $j$ th desired coordinates $(X, Y$, and $Z$ ) and derived coordinates:

$$
\text { Error }_{1}=\sqrt{\left(X_{j}-X_{1}\right)^{2}+\left(Y_{j}-Y_{1}\right)^{2}+\left(Z_{j}-Z_{1}\right)^{2}} .
$$

Eq. (7) shows the constraints for the 3-DOF robot.

$$
l_{2}>d_{1} \geq l_{3}
$$

The desired workspace for the 3-DOF robot ranges from $+20 \mathrm{~cm}$ to $-20 \mathrm{~cm}$ in each of $X, Y$, and $Z$ directions. For a 6-DOF manipulator, Eq. (8) shows its transformation matrix formula:

$$
T_{0}^{6}=T_{0}^{1} \times T_{1}^{2} \times T_{2}^{3} \times T_{3}^{4} \times T_{4}^{5} \times T_{5}^{6} .
$$

Eqs. (9) to (12) present the forward kinematic equations of the 6-DOF robot and the fitness function:

$$
\begin{aligned}
X_{2}= & l_{2}{ }^{*} C_{1}{ }^{*} C_{2}-d_{4}{ }^{*}\left(C_{1}{ }^{*} S_{2}{ }^{*} S_{3}-C_{1}{ }^{*} C_{2}{ }^{*} C_{3}\right) \\
& +l_{5}{ }^{*} S_{5}{ }^{*}\left(S_{1}{ }^{*} S_{4}-C_{4}{ }^{*}\left(C_{1}{ }^{*} C_{2}{ }^{*} S_{3}+C_{1}{ }^{*} C_{3}{ }^{*} S_{2}\right)\right) \\
& +l_{6}{ }^{*} S_{6}{ }^{*}\left(C_{4}{ }^{*} S_{1}+S_{4}{ }^{*}\left(C_{1}{ }^{*} C_{2}{ }^{*} S_{3}+C_{1}{ }^{*} C_{3}{ }^{*} S_{2}\right)\right) \\
& +l_{6}{ }^{*} C_{6}{ }^{*}\left(S _ { 5 } { } ^ { * } \left(S_{1}{ }^{*} S_{4}-C_{4}{ }^{*}\left(C_{1}{ }^{*} C_{2}{ }^{*} S_{3}\right.\right.\right. \\
& \left.\left.\left.+C_{1}{ }^{*} C_{3}{ }^{*} S_{2}\right)\right)-C_{5}{ }^{*}\left(C_{1}{ }^{*} S_{2}{ }^{*} S_{3}-C_{1}{ }^{*} C_{2}{ }^{*} C_{3}\right)\right) \\
& -l_{5}{ }^{*} C_{5}{ }^{*}\left(C_{1}{ }^{*} S_{2}{ }^{*} S_{3}-C_{1}{ }^{*} C_{2}{ }^{*} C_{3}\right), \\
Y_{2}= & l_{2}{ }^{*} C_{2}{ }^{*} S_{1}-l_{5}{ }^{*} C_{5}{ }^{*}\left(S_{1}{ }^{*} S_{2}{ }^{*} S_{3}-C_{2}{ }^{*} C_{3}{ }^{*} S_{1}\right) \\
& -l_{6}{ }^{*} C_{6}{ }^{*}\left(S _ { 5 } { } ^ { * } \left(C_{1}{ }^{*} S_{4}+C_{4}{ }^{*}\left(C_{2}{ }^{*} S_{1}{ }^{*} S_{3}\right.\right.\right. \\
& \left.\left.\left.+C_{3}{ }^{*} S_{1}{ }^{*} S_{2}\right)\right)+C_{5}{ }^{*}\left(S_{1}{ }^{*} S_{2}{ }^{*} S_{3}-C_{2}{ }^{*} C_{3}{ }^{*} S_{1}\right)\right) \\
& -d_{4}{ }^{*}\left(S_{1}{ }^{*} S_{2}{ }^{*} S_{3}-C_{2}{ }^{*} C_{3}{ }^{*} S_{1}\right)-l_{5}{ }^{*} S_{5}{ }^{*}\left(C_{1}{ }^{*} S_{4}\right. \\
& \left.+C_{4}{ }^{*}\left(C_{2}{ }^{*} S_{1}{ }^{*} S_{3}+C_{3}{ }^{*} S_{1}{ }^{*} S_{2}\right)\right)-l_{6}{ }^{*} S_{6}{ }^{*}\left(C_{1}{ }^{*} C_{4}\right. \\
& \left.-S_{4}{ }^{*}\left(C_{2}{ }^{*} S_{1}{ }^{*} S_{3}+C_{3}{ }^{*} S_{1}{ }^{*} S_{2}\right)\right), \\
& +l_{6}{ }^{*} C_{6}{ }^{*}\left(S_{23}^{*} C_{5}+C_{2} 3^{*} C_{4}{ }^{*} C_{5}\right)+l_{5}{ }^{*} C_{23}^{*} C_{4}{ }^{*} S_{6}, \\
Z_{2} S_{5} & +d_{4}{ }^{*} S_{23}+l_{2}{ }^{*} S_{2}+l_{5}{ }^{*} S_{23}^{*} C_{5} \\
& \\
&
\end{aligned}
$$

Eq. (12) is shown in Box II. $X_{2}, Y_{2}$, and $Z_{2}$ are the derived coordinates. $\delta_{2}, \gamma_{2}$, and $\beta_{2}$ (Eqs. (13)

$$
\text { Error }_{2}=\sqrt{\left(X_{j}-X_{2}\right)^{2}+\left(Y_{j}-Y_{2}\right)^{2}+\left(Z_{j}-Z_{2}\right)^{2}+\left(\delta_{j}-\delta_{2}\right)^{2}+\left(\beta_{j}-\beta_{2}\right)^{2}+\left(\gamma_{j}-\gamma_{2}\right)^{2}} .
$$




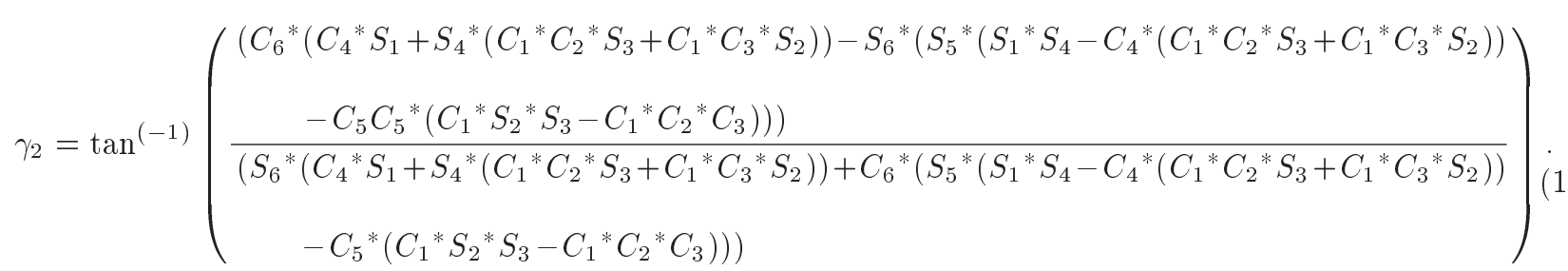

to $(15))$ are the desired orientation of the approach in the workspace of the 6-DOF robot and constraints:

$$
\begin{aligned}
\delta_{2}= & \tan ^{(-1)} \\
& \left(\frac{\left.-\left(S_{6}{ }^{*} S_{23}^{*} C_{5}+C_{2} 3^{*} C_{4}{ }^{*} S_{5}\right)+C_{23}^{*} C_{6}{ }^{*} S_{4}\right)}{\left(S_{23}^{*} S_{5}-C_{23}^{*} C_{4}{ }^{*} C_{5}\right)}\right)
\end{aligned}
$$

Eq. (14) is shown in Box III.

$$
\beta_{2}=-\sin ^{(-1)}\left(C_{6}{ }^{*}\left(S_{23}^{*} C_{5}+C_{23}^{*} C_{4}{ }^{*} S_{5}\right)-C_{23}^{*} S_{4}{ }^{*} S_{6}\right)
$$

Here, $C_{23}$ and $S_{23}$ represent cosine $\left(\theta_{2}+\theta_{3}\right)$ and sine $\left(\theta_{2}+\theta_{3}\right)$, respectively.

\subsection{Fitness function for 6-DOF robot}

\subsubsection{Constraints}

Based on Eqs. (9), (11), (13), and (15), forward kinematics and orientations of $6-\mathrm{DOF}$ are complex and a straightforward solution for link lengths is quite difficult. Hence, GA is used to determine the link lengths for the 6-DOF manipulator to cover the desired workspace. Error 2 shows the equation for the error between all the $j$ th desired coordinates $(X, Y$, and $Z$ ) and orientation of approach (Euler angles $\delta, \beta$, and $\gamma$ ) and derived coordinates and orientation of approach. $\gamma_{2}$ ranges from $+60^{\circ}$ to $-60^{\circ}$. Error $_{2}$ is minimized using GA, as shown in Eq. (12). The transformation equation of the novel 9-DOF manipulator is derived using Eq. (16) [29]:

$$
\begin{aligned}
T_{0}^{10}= & T_{0}^{1} \times T_{1}^{2} \times T_{2}^{3} \times T_{3}^{4} \times T_{4}^{5} \times T_{5}^{6} \times T_{6}^{7} \\
& \times T_{7}^{8} \times T_{8}^{9} \times T_{9}^{10} .
\end{aligned}
$$

Forward kinematic equations and orientation constraints of the novel 9-DOF robotic manipulator are presented by Eqs. (17)-(19):

$$
\begin{aligned}
X_{3}= & l_{1}+d_{3}+d_{8}+d_{9}+l_{4}^{*} C_{7}+l_{6}^{*} C_{7}-d_{5}^{*} S_{7} \\
& -d_{6}^{*} S_{7}+l_{5}^{*} C_{6}^{*} C_{7}, \\
Y_{3}= & l_{3}-l_{7}+d_{2}-l_{8}^{*} C_{8}-l_{9}^{*} C_{8}-l_{10}^{*} C_{8}+l_{4}^{*} S_{7} \\
& +l_{6}^{*} S_{7}+d_{5}^{*} C_{7}+d_{6}^{*} C_{7}+d_{10}^{*} S_{8}+l_{5}^{*} C_{6}^{*} S_{7},
\end{aligned}
$$

$$
\begin{aligned}
& Z_{3}=l_{2}+d_{1}+d_{4}-d_{7}+l_{5}^{*} S_{6}-l_{8}^{*} S_{8}-l_{9}^{*} S_{8} \\
& -l_{10}^{*} S_{8}-d_{10}^{*} C_{8}, \\
& \text { Error }_{3}=\sqrt{\left(X_{j}-X_{3}\right)^{2}+\left(Y_{j}-Y_{3}\right)^{2}+\left(Z_{j}-Z_{3}\right)^{2}} .
\end{aligned}
$$

$X_{3}, Y_{3}$, and $Z_{3}$ are the target coordinates in the workspace of the 9 -DOF robot. The desired workspace for the 6-DOF robot ranges from $+20 \mathrm{~cm}$ to $-20 \mathrm{~cm}$ in each of $X, Y$, and $Z$ directions. Here, $C_{n}$ and $S_{n}$ are cosine and sine of the $n$th joint, respectively, where $n=1,2, \cdots, n$.

\subsection{Fitness function for 9-DOF robot}

\subsubsection{Constraints}

Constraints are added in line with Eqs. (21) to (23):

$$
\begin{aligned}
& \theta_{3}=0, \\
& \theta_{4}=\theta_{7}, \\
& \theta_{5}=-\theta_{6} .
\end{aligned}
$$

These constraints provide the desired orientation of the approach to retrieval of tissue samples while working on a CT machine. Error 3 shows the equation for error between all the $j$ th desired coordinates $(X, Y$, and $Z$ ) and derived coordinates. As the orientation of the robotic 9-DOF manipulator is predefined for the specified tissue retrieval task, the error function in Eq. (20) does not include the orientation segment, as included in Eq. (12) for the 6-DOF manipulator. These equations are used to iterate the GA to find the sets of link length of 3-DOF, 6-DOF, and novel hybrid 9-DOF robotic manipulators. The desired workspace for the 9 -DOF robot ranges from $+20 \mathrm{~cm}$ to $+40 \mathrm{~cm}$ in $X$, $+20 \mathrm{~cm}$ to $-20 \mathrm{~cm}$ in $Y$, and $Z$ directions.

\subsection{Boundry conditions}

Infinite solutions for link lengths and joint displacements are possible for an open chain robotic manipulator system. Hence, boundary conditions need to be set for optimization. Upper and lower boundaries for each robotic manipulator design are shown in Table 4. All dimensions are in centimeter $(\mathrm{cm})$. These boundary 
Table 4. Upper and lower boundaries for 3-DOF, 6-DOF, and 9-DOF robotic manipulators.

\begin{tabular}{|c|c|c|c|c|c|c|c|c|}
\hline \multirow{2}{*}{$\begin{array}{c}\text { Link } \\
\text { parameter }\end{array}$} & \multicolumn{2}{|c|}{ 3-DOF robot } & \multirow{2}{*}{$\begin{array}{c}\text { Link } \\
\text { parameter }\end{array}$} & \multicolumn{2}{|c|}{ 6-DOF robot } & \multirow{2}{*}{$\begin{array}{c}\text { Link } \\
\text { parameter }\end{array}$} & \multicolumn{2}{|c|}{ 9-DOF robot } \\
\hline & Lower & Upper & & Lower & Upper & & Lower & Upper \\
\hline$d_{1}$ & 1 & 15 & $d_{1}$ & 1 & 15 & $d_{3}$ & 1 & 5 \\
\hline$l_{2}$ & 5 & 25 & $l_{2}$ & 5 & 25 & $l_{4}$ & 1 & 15 \\
\hline \multirow[t]{6}{*}{$l_{3}$} & 5 & 25 & $l_{3}$ & 0 & 5 & $l_{5}$ & 5 & 25 \\
\hline & & & $d_{4}$ & 5 & 20 & $l_{6}$ & 1 & 15 \\
\hline & & & $l_{5}$ & 1 & 15 & $l_{7}$ & 1 & 10 \\
\hline & & & $l_{6}$ & 1 & 10 & $d_{8}$ & 1 & 5 \\
\hline & & & & & & $d_{9}$ & 1 & 5 \\
\hline & & & & & & $d_{10}$ & 1 & 5 \\
\hline
\end{tabular}

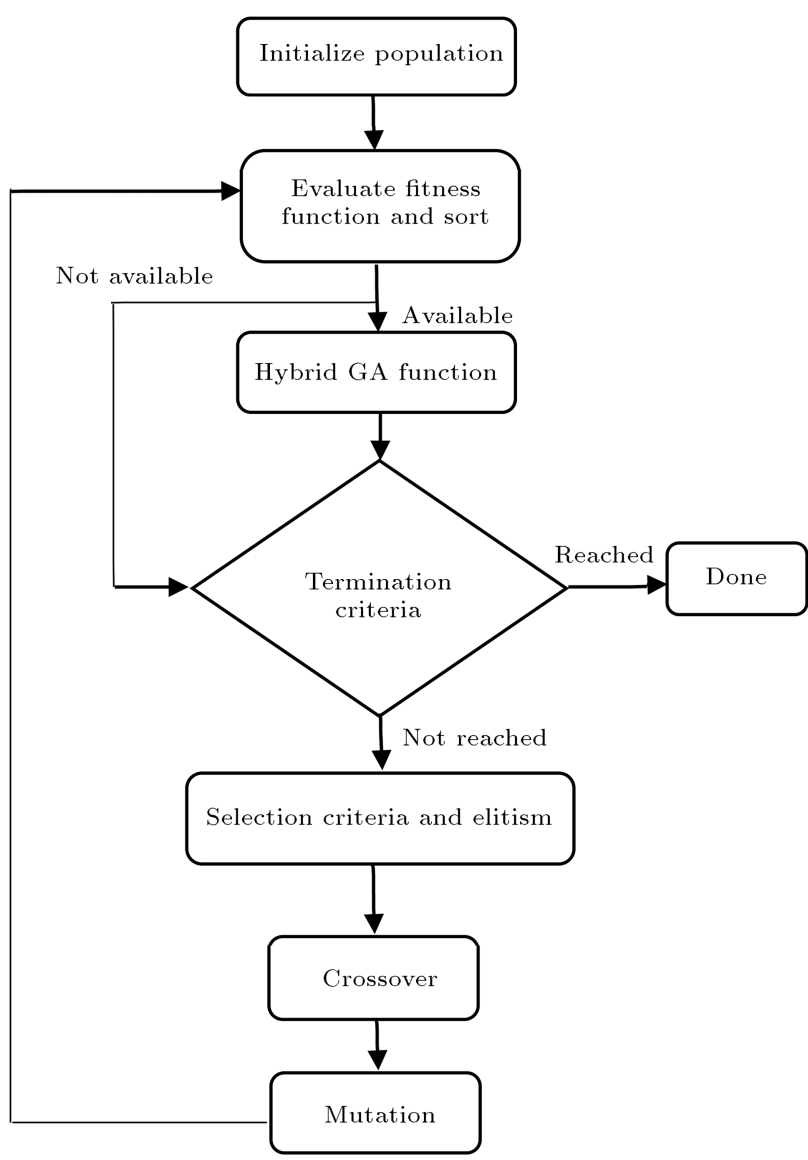

Figure 4. Flowchart representing the process of Genetic Algorithm (GA).

limits are based on the dimensions of the desired workspace.

Using Standard Genetic Algorithm (SGA) and hybrid GA technique, including PS and fmniuc. A flowchart depicting the process of GA is shown in Figure 4.

This process is iterated until a termination criterion is reached. After completing the iterations for SGA, PS, and fmincon hybrid GA, optimum sets of link lengths for all robot manipulators are obtained.

\section{Results and discussion}

GA is trained to obtain the optimum set of link lengths for 3-DOF, 6-DOF, and novel 9-DOF robot manipulators by varying the hybrid functions. Optimization of link lengths and joint displacement is carried out by keeping the rotation angles constant. Hence, link lengths and joint displacement are the variables. The hybrid functions used here are PS and fmincon to minimize the error functions. Firstly, an SGA architecture is trained, which has no hybrid function, and the link lengths are found; then, PS and fmincon are applied. All the other parameters are kept constant. The initial parameters include the creation function, initial population size, initial population range, fitness scaling, selection function, crossover fraction, elite count, mutation function, and constraint algorithm. The termination criteria parameters including the number of generations, stall generation, and function tolerance are also kept constant. The creation function is a nonlinear feasible function with an initial population size of 5 . The initial population size is kept at 5 to reduce the computational cost. The initial population range is kept between -10 and +10 . Fitness scaling is based on the rank system and stochastic uniform is used as the selection function. The crossover fraction is kept at 0.8 and the elite count is 2.5. The mutation function is selected as adaptive feasible and augmented. The Lagrangian function is taken as the nonlinear constraint algorithm. The formula for Lagrange function is shown in Eq. (24):

$$
L=f(x)-\lambda g(x)
$$

The variable $f(x)$ is the fitness function and $g(x)$ the equality function according to Eqs. (19)-(21). The variable $\lambda$ is the Lagrange multiplier which is adjusted while training of the GA.

As for the termination condition, the number of generations is 100 , stall generations are 50, and function tolerance is kept at $10^{-6}$. The link lengths and joint displacements obtained after optimization 


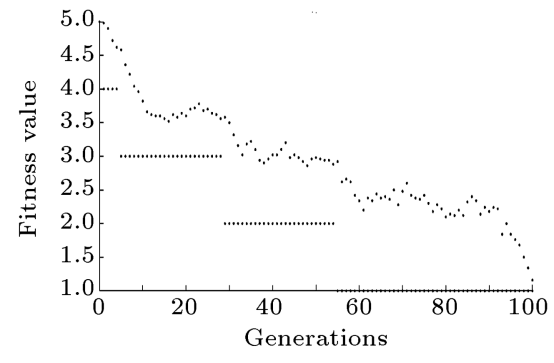

(a)

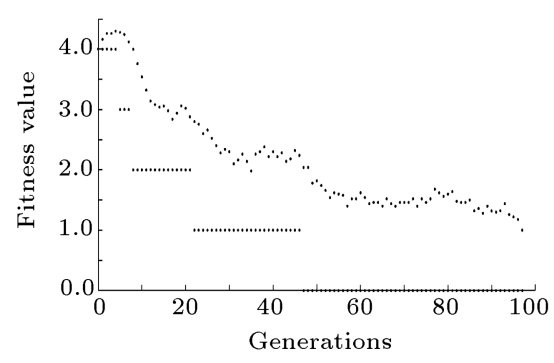

(b)

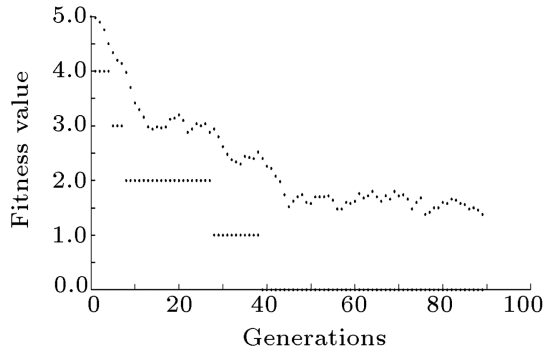

(c)

Figure 5. (a) Training graph of Standard Genetic Algorithm (SGA). (b) Training graph of Genetic Algorithm (GA) with Pattern Search (PS). (c) Training graph of Genetic Algorithm (GA) with fmincon.

Table 5. Link lengths obtained after optimizing the error function using Standard Genetic Algorithm (SGA),

Pattern Search (PS), and fmincon for a 3-DOF robot.

\begin{tabular}{cccc}
\hline $\begin{array}{c}\text { Link } \\
\text { parameter }\end{array}$ & $\begin{array}{c}\text { SGA } \\
(\mathbf{c m})\end{array}$ & $\begin{array}{c}\text { PS } \\
(\mathbf{c m})\end{array}$ & $\begin{array}{c}\text { Fmincon } \\
(\mathbf{c m})\end{array}$ \\
\hline $\mathrm{d} 1$ & 1.00 & 1.00 & 1.00 \\
12 & 20.00 & 20.00 & 14.05 \\
13 & 1.00 & 8.48 & 2.54 \\
\hline
\end{tabular}

will have a sufficient level of accuracy with a tolerance like $10^{-6}$. Even if one decides to manufacture the links of the robotic manipulator, $10^{-6} \mathrm{~cm}$ tolerance provides a sufficient amount of accuracy. These parameters are kept constant throughout the study.

Using the parameters mentioned and training with SGA, PS, and fmincon, the overall fitness value and fitness of each individual in the population of a 3 -DOF robotic manipulator are plotted in Figure 5. Table 5 shows the optimum link lengths of a 3-DOF manipulator, obtained after iteration using all the three functions. According to the graphs, the training reached global minima.

It is observed in Figure 5 that the best overall fitness value for a 3 -DOF robot is obtained using PS while the overall fitness values for SGA and fmincon are 1.16 and 1.38, respectively. Hence, the link lengths determined using PS are optimized better for a 3-DOF robot to reach the desired workspace. The workspaces of this robot are plotted and shown in Figure 6. These workspaces represent the volume covered by the 3-DOF robot with the optimized sets of link length obtained after training the SGA, PS, and fmincon.

Figure 6 shows the desired workspace volume (black) versus the workspace plotted after obtaining the link lengths using SGA (blue), PS (green), and fmincon (red) in two (Figure 6(a)) and three dimensions (Figure 6(b)). It is observed that the SGA produces the workspace closest to the desired one, but the dead zone is very large and, thus, the robot with those link lengths becomes impractical. Dead zones are the regions within the workspace where the end effector of the robotic manipulator cannot be reached. These dead zones result from physical constraints such

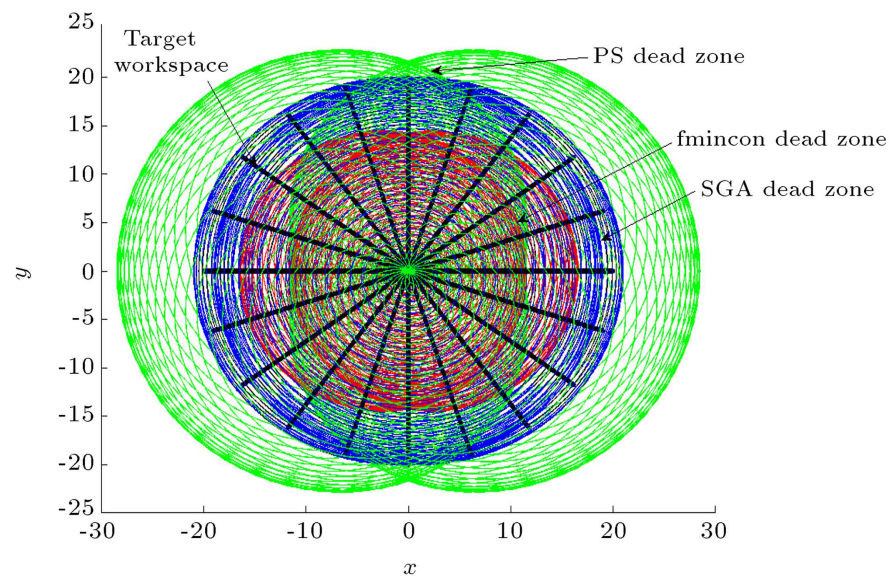

(a)

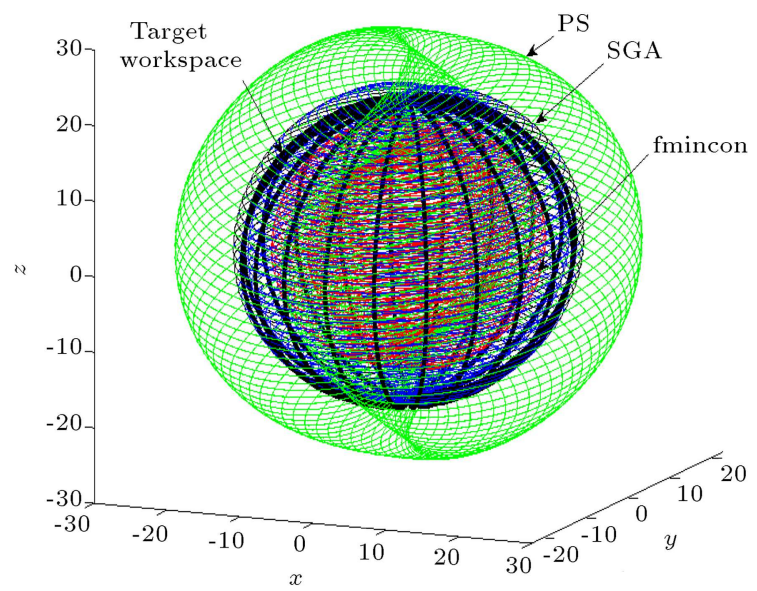

(b)

Figure 6. (a) 2D workspace analysis of 3-DOF robot after using Standard Genetic Algorithm (SGA), Pattern Search (PS) and fmincon. (b) 3D workspace analysis of 3-DOF robot after using Standard Genetic Algorithm (SGA), Pattern Search (PS) and fmincon. 


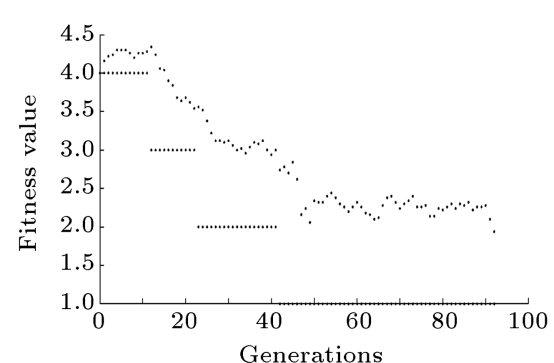

(a)

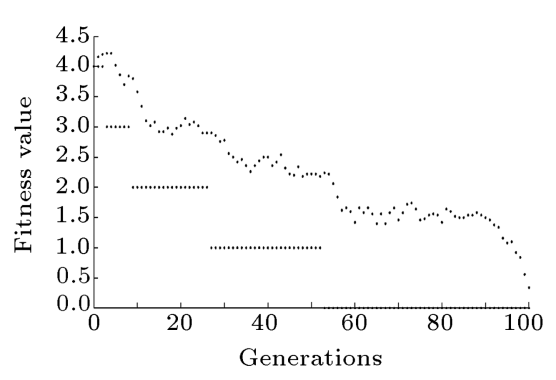

(b)

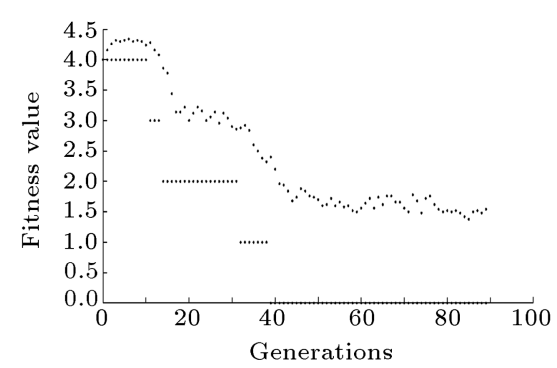

(c)

Figure 7. (a) Training graph of Standard Genetic Algorithm (SGA). (b) Training graph of Genetic Algorithm (GA) with Pattern Search (PS). (c) Training graph of Genetic Algorithm (GA) with fmincon.

as link lengths and joint displacements. On the other hand, the workspace generated by fmincon does not cover the necessary desired volume, while PS provides better results. The entirety of the workspace is covered with the link lengths obtained by PS. Moreover, the dead zone is relatively small. Hence, PS becomes a better choice to obtain optimized link lengths for the 3-DOF robot.

Now, a 6-DOF manipulator is trained using the same parameters and hybrid functions. The overall fitness value and fitness of each individual in the population of a 6-DOF robotic manipulator are plotted in Figure 7. Table 6 shows the optimum lin lengths of a 6-DOF manipulator obtained after iteration using all the three functions. According to the graphs, the training reached global minima.

According to the graphs in Figure 7, the best overall fitness value for a 6 -DOF robot is obtained with PS 0.34, while the overall fitness values for SGA and fmincon are 1.94 and 1.54, respectively. Hence, it is observed that the link lengths found using PS are theoretically more optimized to reach the desired workspace. After training all three GAs, the set of link lengths is obtained and the workspace of the 6-DOF robot using this link lengths is plotted in Figure 8.

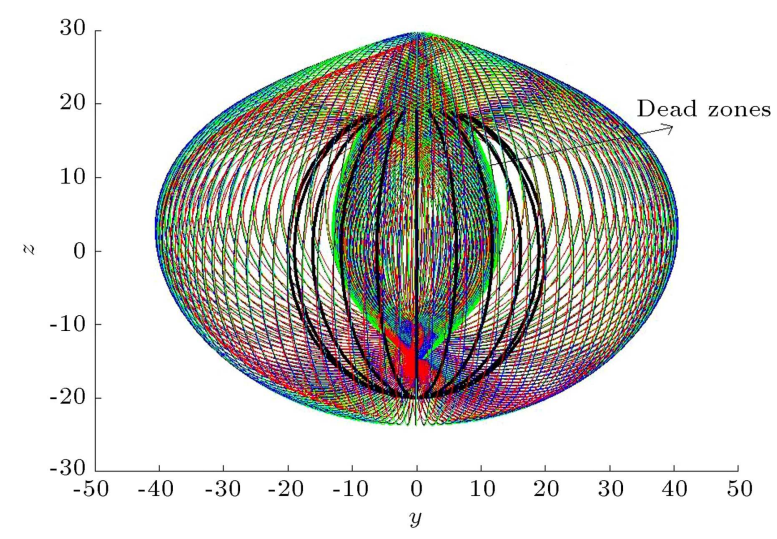

(a)
Table 6. Link lengths obtained after optimizing the error function using Standard Genetic Algorithm (SGA), Pattern Search (PS), and fmincon for a 6-DOF robot.

\begin{tabular}{cccc}
\hline $\begin{array}{c}\text { Link } \\
\text { parameter }\end{array}$ & $\begin{array}{c}\text { SGA } \\
(\mathbf{c m})\end{array}$ & $\begin{array}{c}\text { PS } \\
(\mathbf{c m})\end{array}$ & $\begin{array}{c}\text { Fmincon } \\
(\mathbf{c m})\end{array}$ \\
\hline$d_{1}$ & 3.058 & 3.004 & 3.00 \\
$l_{2}$ & 20.00 & 19.997 & 19.032 \\
$l_{3}$ & 0.412 & 0.101 & 0.610 \\
$d_{4}$ & 13.808 & 13.527 & 12.845 \\
$l_{5}$ & 6.696 & 6.791 & 6.951 \\
$l_{6}$ & 0.166 & 0.117 & 1.289 \\
\hline
\end{tabular}

The constraints and error function ensure that the robotic manipulator reaches the desired position in the workspace at the desired orientation.

Figure 8 shows the workspace analysis of the 6DOF robot with link lengths obtained after optimization using SGA (blue), PS (green), fmincon (red), and desired workspace (black) in two (Figure 8(a)) and three dimensions (Figure 8(b)). It is observed that the volumes covered using SGA and PS are very similar, while the workspace volume obtained by fmincon is slightly smaller with a smaller dead zone.

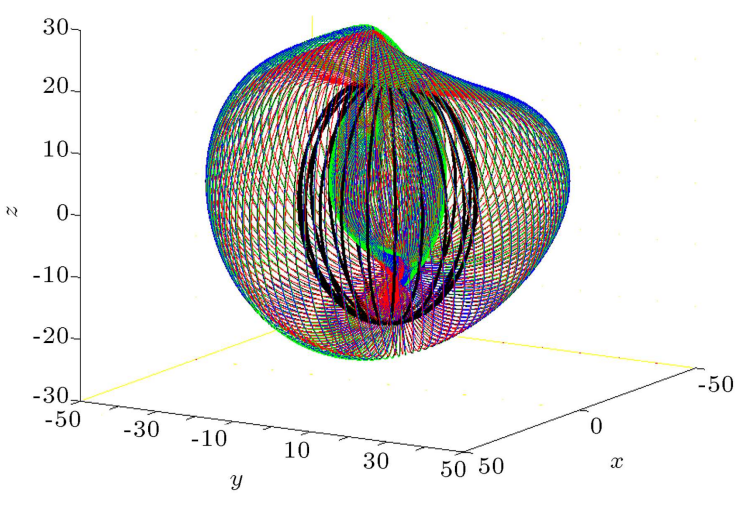

(b)

Figure 8. (a) 2D workspace analysis of 6-DOF robot after using Standard Genetic Algorithm (SGA), Pattern Search (PS) and fmincon. (b) 3D workspace analysis of 6-DOF robot after using Standard Genetic Algorithm (SGA), Pattern Search (PS) and fmincon. 
All the methods cover the desired workspace volume and hence, any function can be applied, but fmicon is more acceptable.

Link lengths for the novel 9-DOF robotic manipulator are optimized using the same parameters and hybrid functions. Here, the desired orientation is fixed as the orientation of approach near the CT machine is limited so as to avoid a collision. The overall fitness value and fitness of each individual in the population of a 9-DOF robotic manipulator are plotted in Figure 9. Table 7 shows the optimum lin lengths of a 9-DOF manipulator, obtained after iteration using all the three functions. It is observed in the graphs that the training reached global minima.

Figure 9 shows that the best fitness value for the novel 9-DOF robot designed for tissue retrieval in a CT environment is found as 0.121 using SGA method. Fitness values for PS and fmincon are 0.124 and 1.36, which are more than those obtained by the SGA method. The set of link lengths obtained from the SGA method should ensure that the robotic manipulator will reach the entire desired target in the desired orientation safely. Lengths of Links 1 and 2 are constant due to $\mathrm{CT}$ bed constraints and arc structure design. The workspace of each set of link lengths
Table 7. Link lengths obtained after optimization using Standard Genetic Algorithm (SGA), Pattern Search (PS), and fmincon for a novel 9-DOF robot.

\begin{tabular}{cccc}
\hline $\begin{array}{c}\text { Link } \\
\text { parameter }\end{array}$ & $\begin{array}{c}\text { SGA } \\
(\mathbf{c m})\end{array}$ & $\begin{array}{c}\text { PS } \\
(\mathbf{c m})\end{array}$ & $\begin{array}{c}\text { Fmincon } \\
(\mathbf{c m})\end{array}$ \\
\hline$d_{1}$ & 40.00 & 40.00 & 40.00 \\
$d_{2}$ & 20.00 & 20.00 & 20.00 \\
$d_{3}$ & 1.00 & 1.002 & 1.00 \\
$l_{4}$ & 9.997 & 9.990 & 6.459 \\
$l_{5}$ & 19.988 & 19.981 & 17.509 \\
$l_{6}$ & 2.012 & 10.693 & 6.317 \\
$l_{7}$ & 5.912 & 5.987 & 4.771 \\
$d_{8}$ & 2.00 & 2.983 & 3.177 \\
$d_{9}$ & 4.970 & 4.993 & 3.867 \\
$d_{10}$ & 4.957 & 2.396 & 2.062 \\
\hline
\end{tabular}

obtained after training all three GA methods is plotted in Figure 10.

Figure 10 depicts the comparison of workspaces plotted after obtaining link lengths using SGA (blue), PS (green), fmincon (red), and the desired target workspace (black) in two (Figure 10(a)) and three dimensions (Figure 10(b)). It is observed that the

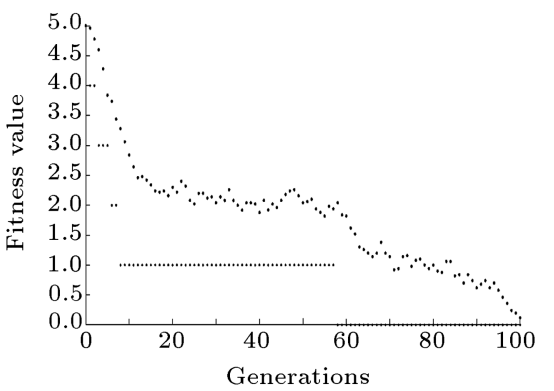

(a)

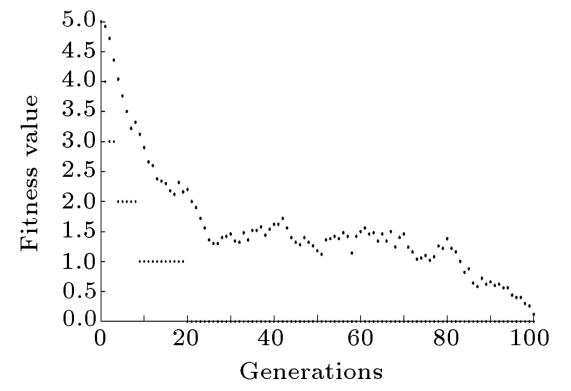

(b)

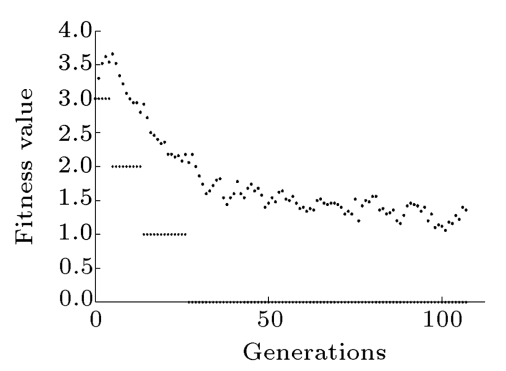

(b)

Figure 9. (a) Training graph of Standard Genetic Algorithm (SGA). (b) Training graph of Genetic Algorithm (GA) with Pattern Search (PS). (c) Training graph of Genetic Algorithm (GA) with fmincon.

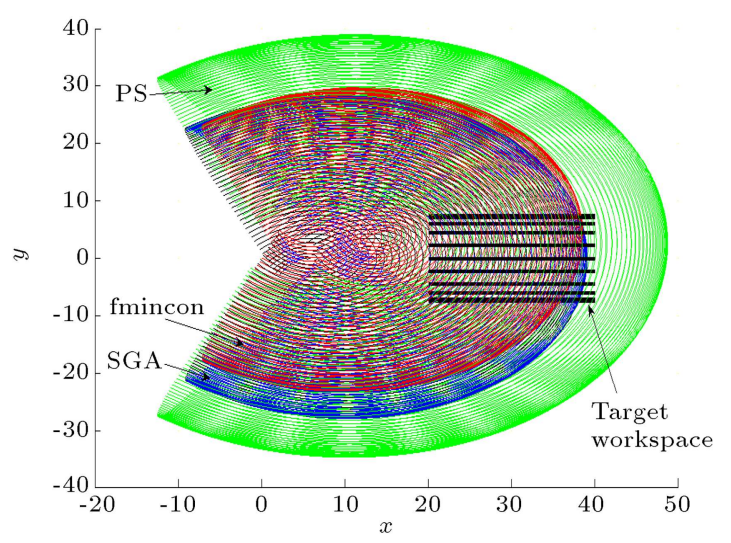

(a)

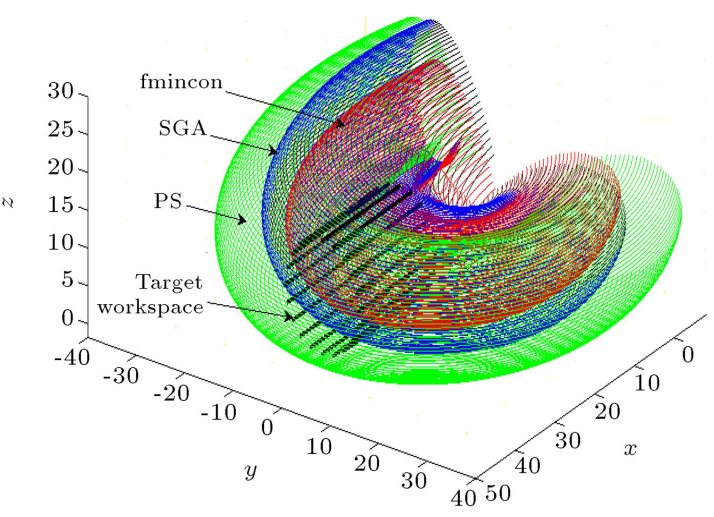

(b)

Figure 10. (a) 2D Workspace analysis of the novel 9-DOF robot manipulator. (b) 3D Workspace analysis of the novel 9-DOF robot manipulator. 
desired workspace is cylindrical in shape as the interior scanning area of a CT machine is cylindrical. The cylindrical workspace is solely targeted on the CT bed and scanning area. The advantage of a cylindrical workspace over a spherical one is that the optimization is focused on the desired CT bed and scanning area. Deadzone is not present within the CT scanning workspace because the optimization is carried out only for the cylindrical workspace. Workspace obtained using PS shows better optimization as all the coordinates of the target workspace are covered. A negligible dead zone is observed in the workspace of the novel 9-DOF robot manipulator. SGA and fmincon cover most of the target workspace, but do not cover some coordinates. Hence, it is observed that the PS hybrid optimization function is useful for finding the link lengths of a robotic manipulator designed for specified tasks under CT machine with only forward kinematic equations and primary constraints.

\section{Conclusion}

In this research, a novel method was proposed to calculate and optimize the $\mathrm{DH}$ parameters including link length and joint displacement of a robotic manipulator with 3-DOF, 6-DOF, and 9-DOF using Standard Genetic Algorithm (SGA), Genetic Algorithm (GA) with Pattern Search (PS), and GA with fmincon hybrid functions. Forward kinematics and error function were used as the fitness function for training the GA and GA with hybrid functions. DH parameters obtained after optimization based on the required workspace were then used for simulating the workspace of the robotic manipulator in MATLAB to observe the workspace and dead-zone coverage. Results demonstrated that in the case of a 3-DOF robot, the GA with PS hybrid function provided better-optimized results as it covered the target workspace with minimal dead zones. For a 6-DOF robotic manipulator, GA with fmincon achieved better results covering the desired workspace in the desired orientation as well. The dead zone for the 6-DOF robotic manipulator after using fmincon was smaller than the other two GA functions. As for the novel 9-DOF robot designed to manipulate a needle in the Computed Tomography (CT) machine environment, GA with PS produced better results. The cylindrical workspace required for this task was covered fully by using the PS hybrid function. With a clear lack of significant research on optimization of $\mathrm{DH}$ parameters for a medical robotic manipulator, the proposed method shows promising results with different Degree Of Freedom (DOF) robot manipulators by minimizing the error function using forward kinematic equations. The only assumptions this method requires are the constraints for the link lengths. Hence, this proposed method can be successfully implemented for finding the optimum link lengths of open chain robotic systems designed to function in the desired workspace.

$\begin{array}{ll}\text { Nomenclature } \\ l & \text { Link length } \\ d & \text { Joint displacement } \\ \alpha & \text { Link twist } \\ \theta & \text { Joint angle } \\ C_{n} & \text { Cosine of } n \text {th joint } \\ C_{23} & \text { Cosine }\left(\theta_{2}+\theta_{3}\right) \\ S_{n} & \text { Sine of } n \text {th joint } \\ S_{23} & \text { Sine }\left(\theta_{2}+\theta_{3}\right) \\ \delta & \text { Euler angle about } X \text { axis } \\ \beta & \text { Euler angle about } Y \text { axis } \\ \gamma & \text { Euler angle about } Z \text { axis } \\ n & \text { Joint number of the robotic } \\ & \text { manipulator }\end{array}$

\section{References}

1. Sun, Y., Liu, H., Luo, Z., and Wang, F. "Robot mechanical structure optimization design", 2007 IEEE International Conference on Robotics and Biomimetics (ROBIO), Sanya, China, pp. 1919-1923 (2007).

2. Albert, F., Koh, S., Chen, C., Tiong, S., and Edwin, S. "Optimizing joint angles of robotic manipulator using genetic algorithm", 2009 International Conference on Computer Engineering and Applications, Singapore, pp. 134-139 (2011).

3. Nearchou, A.C. "Solving the inverse kinematics problem of redundant robots operating in complex environments via a modified genetic algorithm", Mechanism and Machine Theory, 33(3), pp. 273-292 (1998).

4. Situm, Z. and Cikovic, Z. "Optimization of control parameters for servo hydraulic systems using genetic algorithms", Hidravlika-Regulacija, 3, pp. 198-203 (2014).

5. West, C., Montazeri, A., Monk, S., and Taylor, C. "A genetic algorithm approach for parameter optimization of a 7DOF robotic manipulator", IFAC-PapersOnLine, 49(12), pp. 1261-1266 (2016).

6. Kivelä, T., Mattila, J., and Puura, J. "A generic method to optimize a redundant serial robotic manipulator's structure", Automation in Construction, 81, pp. $172-179$ (2017).

7. Chocron, O. and Bidaud, P. "Genetic design of 3D modular manipulators", Proceedings of International Conference on Robotics and Automation, Albuquerque, NM, USA, pp. 223-228 (2021).

8. Jafari, A., Safavi, M., and Fadaei, A. "A genetic 
algorithm to optimum dynamic performance of industrial robots in the conceptual design phase", 2007 IEEE 10th International Conference on Rehabilitation Robotics, Noordwijk, Netherlands, pp. 1129-1135 (2007).

9. Bjorlykhaug, E. and Egeland, O. "Mechanical design optimization of a $6 \mathrm{DOF}$ serial manipulator using genetic algorithm", IEEE Access, 6, pp. 59087-59095 (2018).

10. Saravanan, R., Ramabalan, S., Ebenezer, N.G.R., and Dharmaraja, C. "Evolutionary multi criteria design optimization of robot grippers", Applied Soft Computing, 9(1), pp. 159-172 (2009).

11. Wang, R. and Zhang, X. "Optimal design of a planar parallel 3-DOF nanopositioner with multi-objective", Mechanism and Machine Theory, 112, pp. 61-83 (2017).

12. Francalanza, E., Fenech, A., and Cutajar, P. "Generative design in the development of a robotic manipulator", Procedia CIRP, 67, pp. 244-249 (2018).

13. Bye, R., Osen, O., and Pedersen, B.S. "A computerautomated design tool for intelligent virtual prototyping of offshore cranes", 29th European Conference on Modelling and Simulation, Albena (Varna), Bulgaria, pp. 147-156 (2015).

14. Pires, E., Machado, J., and Oliveira, P. "An evolutionary approach to robot structure and trajectory optimization", Proc. 10th International Conference on Advanced Robotics, Budapest, Hungary, pp. 121-128 (2001).

15. Kazem, B., Mahdi, A., and Oudah, A. "Motion planning for a robot arm by using genetic algorithm", Jordan Journal of Mechanical and Industrial Engineering, 2(3), pp. 131-136 (2008).

16. Mohamed, K., Elgamal, H., and Elsharkawy, A. "Dynamic analysis with optimum trajectory planning of multiple degree-of-freedom surgical micro-robot", Alexandria Engineering Journal, 57(4), pp. 4103-4112 (2018).

17. Kamlesh, S. and Mishra, R. "Advanced path simulation of a $5 \mathrm{R}$ robotic arm for CT guided medical procedures", Materials Today: Proceedings, 5(2), pp. 6149-6156 (2018).

18. Shah, S., Mishra, R., and Mohapatro, G. "Experimental and theoretical design analysis and modeling of a CT image guided robotic arm", 2018 International Conference on Engineering, Applied Sciences, and Technology (ICEAST), Phuket, Thailand, pp. 1-4 (2018).

19. Kumar, P., Sahu, C., and Parhi, D. "Intelligent navigation of a self-fabricated biped robot using a regression controller", Scientia Iranica, 27(1), pp. 262272 (2018).

20. Li, C., Cui, W., Yan, D., Wang, Y., and Wang, C. "Adaptive dynamic surface control of flexible-joint robot with parametric uncertainties", Scientia Iranica, 27(1), pp. 2749-2759 (2018).

21. Soyaslan, M., Uk, M., Ali Shah, F., and Eldogan, O. "Modelling, control and simulation of a SCARA PRRtype robot manipulator", Scientia Iranica, 27(1), pp. 330-340 (2018).

22. Ma, J., Liu, Y., Zang, S., and Wang, L. "Robot path planning based on genetic algorithm fused with continuous bezier optimization", Computational Intelligence and Neuroscience, 2020, pp. 1-10 (2020).

23. Baressi Šegota, S., Anđelić, N., Lorencin, I., Saga, M., and Car, Z. "Path planning optimization of six-degreeof-freedom robotic manipulators using evolutionary algorithms", International Journal of Advanced Robotic Systems, 17(2), pp. 172-182 (2020).

24. Cooper, P., Griffiths, C., Andrzejewski, K., and Giannetti, C. "Motion optimisation for improved cycle time and reduced vibration in robotic assembly of electronic components", AIMS Electronics and Electrical Engineering, 3(3), pp. 274-289 (2019).

25. "Equipment", Department of Radiology, 2021. [Online]. Available: https://radiology.wisc.edu/research/ modalities/ct/equipment/.

26. Taylor, C. "Adaptation in natural and artificial systems: An introductory analysis with applications to biology, control, and artificial intelligence. Complex adaptive systems. John H. Holland", The Quarterly Review of Biology, 69(1), pp. 88-89 (1994).

27. Mittal, R. and Nagrath, I. "Manipulator kinematics", In Robotics and Control, 1st Ed., New Delhi, India: Tata McGraw-Hill, 2003, p. 222 (2003).

28. Ge, X. "Evaluating and optimization of 7-DOF automated fiber placement robotic manipulator performance index based on AdaBoost algorithm", Journal of Computational Methods in Sciences and Engineering, 18(3), pp. 749-757 (2018). Available: $10.3233 / \mathrm{jcm}-180826$.

29. Fahim, S., Sarker, Y., and Sarker, S. "Modeling and development of a five DoF vision based remote operated robotic arm with transmission control protocol", SN Applied Sciences, 2(7), 1263 (2020).

\section{Biographies}

Shubham Kamlesh Shah is a PhD Research Scholar at School of Mechanical Engineering, KIIT deemed to be University. He specialized in robotics, machine learning, and machine design. He completed his BSc and MSc in Technology in Mechanical Engineering and Machine Design from KIIT deemed to be University, Bhubaneswar, Odisha India. He has published many research articles in international journals and conferences. He also has three national and international patents published.

Ruby Mishra received BE (Mechanical Engineering) 
from Utkal University in 1999, MTech (Machine Design) from Jadavpur University in 2007, and PhD (Machine Design) from Jadavpur University in 2014. She has 21 years of teaching and research experience. Her research interests include kinematics of machine and mechanism, biomedical engineering, biomedical signal and image processing, robotics, and medical image analysis. She is a member of the Institute of Engineers and Indian Science Congress Association. She has published 3 national and international patents. She has published many article papers that are indexed in Scopus, Web of Science, and Google Scholar. Currently, she is doing research on biomedical instruments like robotic arm for CT Guided Biopsy and Single Lumen Microcatheter for Angiography and Embolization. At present, she is working as an Associate Professor at the Mechanical Engineering Department of KIIT deemed to be University. 